\title{
SCIENCE DE L'HOMME ET DIVISION DES SCIENCES SELON MAINE DE BIRAN
}

\author{
François Azouvı
}

\begin{abstract}
RÉsume : Comme beaucoup de ses contemporains, Maine de Biran s'attache tout au long de sa carrière philosophique à élaborer une "science de l'homme ". Mais l'originalité de la science biranienne de l'homme est d'être construite selon une perspective résolument épistémologique. Il y a autant de sciences dans la science de l'homme qu'il y a de "points de vue" pour l'esprit; chacun détermine un "ordre de faits ", se déploie selon une méthode propre, atteint des résultats spécifiques et rencontre des obstacles particuliers. La division des sciences est donc la tâche qui prélude à l'analyse de chacune d'elles; c'est elle qui occupe Maine de Biran à chacun des moments clés de son itinéraire.
\end{abstract}

" L'idéologie plane, pour ainsi dire, sur toutes les sciences, car les sciences ne se composent que de nos idées et de leurs divers rapports. Ces idées forment comme un pays immense et infiniment varié, partagé en une multitude de districts, coupés par un plus grand nombre de routes de communication. Pendant que les savants voyageurs se dispersent dans ces districts, [...] l'idéologiste, placé sur une éminence et comme immobile, observe leurs directions, en tient note, en dresse la carte ${ }^{1}$.

Le jeune « idéologiste » qui, à la fin de 1802, écrit ces lignes dans une note Sur les rapports de l'Idéologie et des mathématiques, est aussi, de l'aveu de Cabanis qui la lui a commandée, un "géomètre distingué " ${ }^{2}$ dont on attend qu'il contribue à l'Idéologie nouveau-née en la dotant de cette «carte" des sciences qui manque encore. Mais l'idéologiste va bientôt devenir Maine de Biran; au début de 1804 exactement, au moment où il travaille d'arrache-pied au Mémoire sur la décomposition de la pensée qui sera couronné par l'Institut, le 8 mars 1805 . Nul doute

1. Mémoires sur les rapports de l'Idéologie et des mathématiques, in MAINE de BirAN, Euvres, éd. François Azouvi, Paris, Vrin, 1988, t. III, p. 8. Cette édition sera désormais désignée par la lettre A, suivie du numéro du tome et de celui de la page.

2. Ibid., A, III, 1.

Revue de synthèse: IV S. N ${ }^{\circ} 1-2$, janv.-juin 1994. 
qu'en devenant lui-même, Biran décevra ses mentors : la carte des sciences à laquelle Cabanis ou Tracy pouvaient songer ne sera pas faite par lui ; il ne sera pas le continuateur de Bacon et de D'Alembert, l'artisan d'une division encyclopédique des sciences réévaluée en fonction des disciplines nouvelles, surgies entre-temps. C'est sur une critique de la conception baconienne que s'ouvrira la Décomposition de la pensée, comme si l'ex-idéologue avait à cœur de marquer d'entrée de jeu, sur cette question cruciale, l'importance du pas franchi : " L'immortel restaurateur de la vraie philosophie, Bacon, embrassant dans un système général toutes les connaissances humaines et dessinant l'arbre de leur généalogie $[\ldots]{ }^{3}$ : voilà exactement ce que Biran ne fera pas.

Mais on peut dire aussi, à l'inverse, qu'il ne fera rien d'autre durant les vingt années qui lui restent à vivre, que travailler à cette question de la division des sciences dont il esquissait le projet en 1802. À la condition toutefois d'ajouter deux précisions : d'une part, la façon dont il détermine la question et les réponses qu'il lui apporte introduisent, entre sa doctrine et celle de la tradition baconienne, un écart si considérable que l'on ne peut pas voir dans celle-là l'héritage de celle-ci ; d'autre part, la question générale de la «carte » des sciences disparaît chez lui au profit d'une question particulière : la carte de la science de l'homme.

Examinons d'abord ce second point. Avant de se montrer intéressé aux rapports de l'Idéologie et des mathématiques, Maine de Biran est quelqu'un qui a lu dans les Nouveaux éléments de la science de l'homme de Barthez, ces mots fameux : « La science de l'homme est la première des sciences [...] elle a fait peu de progrès jusqu'à présent, et même beaucoup moins à proportion que n'en ont fait d'autres sciences utiles $"{ }^{4}$. Le propos tombe dans une oreille bien faite pour l'entendre : Biran est le premier concerné par l'avancement d'une science dont il y a lieu d'espérer qu'elle l'aidera à déchiffrer l'énigme qu'il est à ses propres yeux. « Ce n'est pas sur des sujets étrangers qu'il faut expérimenter, note-t-il en 1794 ou 1795 , c'est sur soi-même ${ }^{5}$. De quelles expériences s'agit-il ? Il s'agit de « tenir registre de ses sentiments particuliers ", d'en observer les variations selon les variations de l'état physique, du climat, de l'âge, de la santé, des modes de la sensibilité, etc. Si ce travail était fait, on aurait autant de mémoires que d'observateurs d'eux-mêmes, et alors, " quelle lumière rejaillirait sur la science de l'homme $!{ }^{6}$. Maine de Biran, toute

3. Mémoire sur la décomposition de la pensée, A, III, 19.

4. Paul-Joseph Barthez, Nouveaux éléments de la science de l'homme, Paris, 1778, p. I.

5. Maine de Biran, Journal, éd. Henri Gouhier, Neuchâtel, La Baconnière, 1957, t. III, p. 18 .

6. Ibid., p. 10 . 
sa vie, restera "étonné de se sentir exister " ${ }^{7}$ : si la science de l'homme est sa grande préoccupation, c'est parce que, d'elle, il attend qu'elle dissipe l'étonnement.

Aussi n'en abandonnera-t-il jamais l'analyse. Depuis les fragments autobiographiques de 1794-1795 jusqu'à l'anthropologie de 1824, le problème posé par la constitution d'une science de l'homme qui soit à la fois complète et philosophiquement consistante, ne disparaît à aucun moment de son horizon. Il est présent durant la période 1804-1812, au cours de laquelle Biran élabore ce que l'on pourrait appeler, pour faire court, une psychologie phénoménologique dont l'exposé le plus achevé est l'Aperception immédiate (1807); il est présent autour de 1813-1815, lorsque le philosophe s'efforce de doter cette psychologie d'une ontologie; il est présent enfin lorsque la science de l'homme prend le nom d' " anthropologie ", à partir de 1818-1819, et qu'elle culmine dans une "vie de l'esprit " dont le "géomètre distingué " de 1802 n'avait certes pas idée.

Mais si Maine de Biran travaille vingt ans durant à la constitution d'une " carte " de la science de l'homme, c'est qu'il en conçoit l'établissement d'une telle manière qu'aucun de ses illustres prédécesseurs ne peut lui servir d'exemple. Ni Bacon, quels que soient ses mérites, ni D'Alembert, ni des modernes comme Barthez, ne sont parvenus, selon lui, à une détermination vraiment fondamentale des principes sur lesquels doit reposer la théorie philosophique de la science de l'homme. La raison de cet échec est donnée, une fois pour toutes, au début de la Décomposition de la pensée : Bacon, et ses disciples après lui, est parti de la division encyclopédique elle-même et, remontant des produits à leurs causes supposées, en a déduit la division des facultés de l'âme en raison, mémoire, imagination ${ }^{8}$. Loin d'être fondatrice, la division des facultés est demeurée seconde et hypothétique; celle des sciences ne pouvait donc à son tour qu'être conventionnelle.

Cette critique ne prend évidemment son sens qu'à la lumière de la thèse biranienne elle-même. Quand et où celle-ci apparaît-elle? Dans les textes mêmes où surgit la doctrine de l'effort, textes qui marquent, pour reprendre la terminologie forgée par Henri Gouhier, la conversion de Biran au biranisme ${ }^{9}$ : dans les lettres du printemps 1804 à Destutt de

7. Méditation sur la mort près du lit funèbre de sa sour Victoire, in MAINE de Biran, Euvres, éd. Tisserand (édition désormais désignée par T, suivie du numéro du tome et de celui de la page), Paris, Alcan, 1920, p. 12. Pour une occurrence tardive de la même formule, cf. Journal, op. cit. supra n. 5, t. II, p. 399.

8. Mémoire sur la décomposition de la pensée, A, III, 303. Cf. aussi Essai sur les fondements de la psychologie, T, VIII, 46.

9. Henri Gounier, Les Conversions de Maine de Biran, Paris, Vrin, 1947. C'est le titre du chap. III. 
Tracy. La chose mérite d'emblée d'être notée, car elle montre bien que la découverte de la méthode susceptible de fonder la science de l'homme est inséparable de la découverte du biranisme et qu'elle est au cœur de cette philosophie.

On ne fera pas ici l'analyse de cette correspondance; on se bornera à en rappeler le contexte. Maine de Biran a lu en novembre 1798 le Mémoire sur la faculté de penser de Destutt, publié dans le tome I des Mémoires de l'Institut national; c'est de cette lecture qu'il dira plus tard qu'elle fit dans son esprit une "révolution " telle, que plus rien après ne fut comme avant ${ }^{10}$. Dans le Mémoire de Destutt, Biran trouve ce qui manquait à Condillac : le rôle de la motricité dans la genèse de l'extériorité. Comme l'on sait, les thèses du Mémoire passent dans le premier volume des Éléments d'Idéologie, qui parait en 1801 sous le titre : Projet d'éléments d'Idéologie. Biran en prend connaissance tout de suite, mais découvre " avec étonnement et une sorte de chagrin " que Tracy est infidèle à sa propre découverte : pour résoudre une difficulté qu'il aperçoit dans sa doctrine de 1798, il forge en 1801 une solution qui oblige Biran à se demander si l'auteur du Mémoire sur la faculté de penser a parfaitement aperçu la profondeur de ses vues. En 1798, Tracy pensait que la sensation de mouvement arrêté par un obstacle suffisait à engendrer la conscience d'extériorité. En 1801, il estime que le mouvement n'est instructif que s'il est volontaire; il ajoute donc la volonté aux requisits de la genèse de l'extériorité. Mais alors, la volonté ne peut naitre du mouvement; elle doit le précéder et préexister en l'individu. D'où la façon dont il la définit en 1801 : la volonté est la " faculté que nous avons de sentir ce qu'on appelle des désirs ", elle est une "conséquence immédiate et nécessaire " de certaines sensations ${ }^{11}$, un " résultat de notre organisation ${ }^{12}$.

Biran lit les Éléments d'Idéologie et relit le Mémoire sur la faculté de penser : si la volonté est ce qu'en disent les Éléments, elle ne peut jouer le rôle dévolu à la motricité dans le Mémoire. D'où vient ce flottement dans la pensée de Tracy? Au moment où Biran peut répondre à la question, il est en possession de sa méthode, celle qui va présider à l'élaboration de la "carte" de la science de l'homme. "Dans un corps de doctrine", écrit-il au père de l'Idéologie, « tout doit être homogène depuis le premier point de départ jusqu'aux conséquences, déductions ou applications les plus éloignées ${ }^{13}$. "Quel est le point de départ? C'est l'idée première

10. A Destutt de Tracy, printemps 1804, T, VII, 230.

11. Antoine Destumt de Tracy, Éléments d'Idéologie, t. I, éd. H. Gounier, Paris, Vrin, 1970 , p. 67.

12. Ibid., p. 68.

13. A Destutt de Tracy, printemps 1804, T, VII, 250-251. 
sous laquelle nous concevons le sujet sentant et pensant. " On ne saurait trop insister sur l'importance de cette phrase et de celles qui suivent : car c'est le lieu d'apparition de la doctrine biranienne des "points de vue " qui détermine toute sa théorie de la connaissance; l'homme peut être considéré sous différents points de vue qui sont autant de catégories auxquelles doit se conformer la série entière des propositions. Quels sont les différents points de vue? La liste qu'ébauche ici Biran n'est encore ni très solide ni très nette; mais elle indique au moins que le principe est trouvé :

" cette idée [première] peut être simplement réfléchie, c'est-à-dire fondée sur l'observation de nous-mêmes. On peut enfin chercher à la baser sur un point de vue en quelque sorte extérieur à notre être actuel, déterminer par exemple les conditions réelles, instrumentales [...] qui peuvent constituer un sujet capable de sentiment. "

Point de vue de l'expérience intérieure, point de vue de l'expérience extérieure : la grande opposition, celle qui traverse et gouverne toute l'œuvre de Biran et qui partage le champ du connaissable en deux modalités hétérogènes, est saisie là in statu nascendi. Il y a deux sortes de connaissances, donc "deux ordres de faits » et deux types de lois, parce qu'il y a deux catégories pour les produire. Non pas tant deux régions, ou deux domaines - pareil contresens sur la théorie biranienne de la connaissance a longtemps obéré sa juste appréciation -, mais deux modes de production des connaissances : l'un, par réflexion ou aperception, grâce auquel le sujet détermine ce que sont les choses pour lui, sujet connaissant; l'autre, par représentation, où il détermine ce que sont les choses objectivement, abstraction faite du sujet pour qui elles sont.

Aussitôt énoncée, la méthode est appliquée à la science de l'homme dont elle va tracer les contours. Les " points de vue " donnent lieu à des connaissances aussi valides les unes que les autres, mais qui diffèrent dans leurs "procédés" comme dans les "résultats" auxquels ils conduisent. On devine que deux conséquences, fondamentales toutes deux, vont en résulter. D'une part, « on peut s'en servir tour à tour et les faire converger jusqu'à un certain point à la connaissance la plus exacte possible des diverses parties qui composent la science de l'homme ". On peut est évidemment une formule maladroite; car Biran pense on doit ; et plus exactement : on doit parce qu'on peut. La science de l'homme ne fera en effet les progrès qu'attendait d'elle Barthez, qu'en menant les connaissances selon les deux axes offerts par les points de vue. Si la science de l'homme est si longtemps restee dans les limbes, c'est faute d'avoir compris qu'elle devait associer les résultats des sciences spéciales. 
Mais, d'autre part, cette association ne peut se faire ingénument; c'est la seconde conséquence qui résulte de la règle des points de vue :

« il est dangereux de ne pas bien distinguer les idées qui se trouvent formées d'après l'une ou l'autre méthode, de vouloir combattre les unes par les autres, enfin de prétendre les opposer ou les concilier dans l'explication d'une seule et même espèce de phénomènes. "

En d'autres termes : une thèse de physiologie, par exemple, ne peut ni contredire ni prouver une thèse de psychologie; quoiqu'elles se servent souvent « des mêmes mots ", elles ne désignent pas les mêmes choses.

Dans la perspective qui est celle de la correspondance avec Tracy, la découverte de la règle de méthode donne sens aux flottements du maître. La contradiction entre les deux acceptions du mouvement volontaire vient de ce que celui-ci les a prises dans l'un puis dans l'autre point de vue, sans réaliser qu'il en changeait et en les traitant par conséquent comme si elles étaient sur le même plan. Dans le point de vue de la réflexion, la volonté ne peut pas préexister au mouvement qu'elle est; elle est un commencement. Dans le point de vue de l'objectivité, il est exact qu'il y a des conditions à la naissance de la volonté, que des désirs la précèdent et sont précédés eux-mêmes par des mouvements instinctifs. Cabanis et Bichat fournissent là-dessus des explications fort utiles, et d'autant mieux utilisables par Biran qu'il sait désormais comment entendre la leçon qu'elles délivrent : les analyses physiologiques de ces maîtres en "idéologie objective ${ }^{14}$ fournissent des renseignements sur la façon dont se fait la genèse du sujet, mais pour un tiers, et non pas pour le sujet lui-même. Dans le point de vue de Cabanis et de Bichat, le sujet " ne pourra être dit avoir conscience de son existence; il sera ou existera sentant pour les autres, et non point pour lui-même ${ }^{15}$. La division des sciences, fondée sur l'hétérogénéité des points de vue, permet que soient vraies en même temps, mais pas dans le même ordre de faits, des propositions contradictoires comme celles-ci : la volonté est un commencement radical ; la volonté est précédée par des mouvements involontaires.

Les lettres à Destutt du printemps 1804 offrent donc la primeur de la découverte par Biran de sa méthode et de sa doctrine, inséparables l'une de l'autre. Mais elles posent plus de questions qu'elles n'en résolvent : comment parvient-on à la détermination de ces points de vue, au moyen de quelle « décomposition » de la pensée ? Quelle est la relation entre les points de vue et les facultés ? Est-on sûr qu'il n'y en a que deux ? Comment détermine-t-on les sciences auxquelles donnent lieu les points de

14. A Destutt de Tracy, printemps 1804, T, VII, 238.

15. Ibid., T, VII, 250. 
vue? Toutes ces questions ne sauraient être traitées dans le cadre du présent travail. Je m'attacherai aux deux dernières, en me contentant de donner pour les deux premières une indication brève : la détermination des facultés qui fondent les points de vue, lesquels, à leur tour, donnent les deux ordres de faits, s'opère dans la Décomposition de la pensée et dans les autres textes par une analyse régressive. En gros, la doctrine de Biran est sur ce point constante.

La question du nombre de points de vue et celle des sciences qui composent la science de l'homme est plus malaisée. Il n'y a aucun doute sur le fait que, de 1804 jusqu'à la fin, Maine de Biran conçoit deux ordres de faits et deux seulement. Depuis la Décomposition de la pensée jusqu'aux Considérations sur les principes d'une division des faits psychologiques et physiologiques (1823) et aux Nouveaux essais d'anthropologie (1824), il va répétant que l'expérience est scindée en deux, qu'il y a deux modes opposés de connaitre. L'erreur à ne pas commettre serait de croire que cette division fait que chacune des sciences est partielle parce qu'elle a en charge une partie de l'expérience. C'est bien en quoi la doctrine biranienne n'est pas une psychologie des facultés. Parce que la faculté et le point de vue sont une " idée première " et non pas une aptitude, l'ordre de faits dont ils produisent la connaissance n'a rien d'incomplet. « Les deux ordres de faits sont complets chacun en son genre, sans que l'un paraisse rien ajouter à l'autre ${ }^{16}$. La chose est évidemment d'importance. Car il en résulte que chaque science est parfaitement autonome, qu'elle n'a pas à être limitée ou à recevoir une juridiction d'une autre. Pour le naturaliste qui s'occupe de l' "homme extérieur", il est normal que celui-ci soit tout l'homme. Autrement dit, il ne manquera rien à la physiologie si elle n'inclut pas les considérations tirées de la psychologie et viceversa. Cela n'empêche pas, en revanche, que la science de l'homme soit incomplète si elle ne contient que l'une des deux sciences. Complètes chacune en leur genre, la science des faits extérieurs et celle des faits intérieurs demandent à être associées pour qu'à son tour, la science de l'homme soit complète dans le sien.

La division biranienne des points de vue, sur laquelle repose celle des sciences, a donc ceci de particulier qu'elle fait de l'ignorance par chacune des résultats ou des hypothèses de l'autre, non seulement quelque chose de possible mais quelque chose de normal. Si la phrénologie de Gall, par exemple, est inacceptable, c'est d'une part en raison des problèmes strictement scientifiques qu'elle pose - anatomiques et physiologiques -, mais aussi en raison des " incursions " qu'elle fait, sans le savoir, dans le domaine de la psychologie. Biran reproche à Gall sa théorie de la locali-

16. Nouveaux essais d'anthropologie, A, X-2, 20. 
sation cérébrale parce qu'elle procède, en l'absence d'un critère anatomophysiologique rigoureux, d'une division des facultés dont seule la conscience peut avoir livré la clef. Semblablement, il reproche à Cabanis sa fameuse phrase sur la sécrétion de la pensée, parce qu'elle implique la mise en relation entre un ordre de faits accessibles à l'expérimentation scientifique et un ordre de faits dont la conscience est juge. Si vous avez raison, dit-il à l'auteur des Rapports $d u$ physique et $d u$ moral, montreznous la substance produite par la sécrétion; si vous ne le pouvez pas, c'est bien qu'elle est d'un autre ordre que celui dont vous prétendez la dériver, et vous ne pouvez pas assigner un rapport de production entre ces deux ordres. Le matérialisme n'est pas, pour Biran, une fausse doctrine scientifique, mais une doctrine qui mélange les points de vue et qui est, pour cette raison et non pas pour les thèses qu'elle professe, non valide. La doctrine inverse, celle qui étend les notions psychologiques à la physiologie, est au même titre une erreur.

En droit, il devrait y avoir autant de points de vue qu'il y a d'ordres de faits et donc de sciences au sein de la science de l'homme; Maine de Biran se tient-il fermement à ce droit? Les choses sont moins simples. Un texte du Mémoire sur la décomposition de la pensée oblige à réviser la conception binaire que $\mathrm{j}$ 'ai dite.

" Il y a un sentiment immédiat, absolu et général de tous les éléments sensibles unis dans une même combinaison vivante, il y a une aperception individuelle qui a pour objet distinct (aussi immédiat) l'ensemble des parties obéissantes à la même volonté et pour sujet un celui de l'effort (relatif) même, employé à les diriger, à les contracter, à les mouvoir; enfin, il y a une représentation tout à fait objective de ces instruments ou moyens de motilité, telle que se la font les anatomistes ${ }^{17}$.

Que ces trois catégories - le sentiment immédiat, l'aperception et la représentation - soient bien des points de vue, c'est ce qui résulte de la recommandation qui suit aussitôt : " Il n'est pas permis de confondre, dans aucun cas, ces trois points de vue distincts sur lesquels s'appuie la science de l'homme. "

Ni l'aperception ni la représentation ne posent de problème vraiment délicat. La première préside toujours à cette science que Maine de Biran appelle, autour de 1805 , « idéologie subjective " ${ }^{18}$, et à partir de 1807 , "psychologie ». Parmi tant d'autres, retenons cette façon de la définir, comme une science qui, " cherchant à remonter jusqu'à l'origine du moi ou de la personnalité humaine, part de là pour explorer le vaste et majes-

17. Mémoire sur la décomposition de la pensée, A, III, 126.

18. Ibid., A, III, 25-60. 
tueux ensemble de phénomènes où ce moi assiste tantôt comme témoin et tantôt comme acteur ${ }^{19}$. La psychologie est la science subjective du sujet. La "représentation" préside, d'une façon large, aux sciences objectives du sujet. Le texte de la Décomposition mentionne l'anatomie; mais les Rapports disent plus généralement que c'est le point de vue du

« naturaliste qui s'attache à l'homme extérieur, ne le considère que dans son enveloppe matérielle et à sa surface, par les côtés sous lesquels il se trouve, par sa capacité réceptive d'impressions, en dépendance nécessaire de tout ce qui l'environne $"{ }^{20}$.

Un autre passage, dans l'Essai, a le mérite de distinguer les sciences qu'inclut cette catégorie large, et de le faire dans le cadre d'une déduction rigoureuse. Dépendantes de la coordination des phénomènes dans l'espace, on trouve, dit Biran, l'histoire naturelle, la botanique, l'anatomie, la géologie, la géographie, la cosmographie et l'hydrographie. Le propre de ces sciences d'observation est qu'il n'y a « rien à expliquer » en elles, parce qu'il n'y a " rien à déduire d'une cause " ${ }^{21}$. Le processus de la science commence à l'observation, et s'arrête à la classification des phénomènes : on a tout dit d'une chose lorsqu'on a montré à quelle place il faut la ranger. Les sciences qui impliquent la coordination des phénomènes dans le temps sont les sciences physiques et physico-mathématiques; parmi les premières, la physiologie animale ou végétale, la physique, la mécanique, l'hydrodynamique, etc. Parce que leurs phénomènes se succèdent dans un temps et paraissent liés les uns aux autres, l'observation et la classification ne sont que les deux premières étapes d'une démarche qui vise à l'établissement des causes et à la détermination des lois.

Lesquelles de ces sciences concernent-elles la science de l'homme? Parmi les sciences de la coordination dans l'espace, Biran ne mentionne que l'anatomie. Prenons-le comme un fait : l'histoire naturelle ne fait pas partie des disciplines au concours desquelles la science de l'homme doit d'être complète. En revanche, l'anatomie y figure de plein droit. C'est elle qui, notamment, sert à décrire les organes du système musculaire; c'est elle qui est convoquée pour offrir une base rigoureuse aux discussions sur la sensorialité, et particulièrement pour la question des affections inconscientes; c'est sur le terrain de l'anatomie que reposent d'abord les questions controversées du siège de l'âme, du système nerveux et du point de réunion des nerfs. Mais c'est surtout à la physiologie,

19. Appendice XVII, A, V, 167.

20. Rapports des sciences naturelles avec la psychologie, A, VIII, 25.

21. Essai sur les fondements de la psychologie, T, VIII, 570-571. 
science de la coordination des phénomènes dans le temps, que renvoie Maine de Biran lorsqu'il parle de la connaissance objective de l'homme. Sur ce point, il faut évidemment s'arrêter un instant.

Pourquoi la physiologie ? Ou si l'on préfère, pourquoi pas la physique? La question mérite d'autant plus d'être posée, qu'en deux endroits au moins, Maine de Biran procède à une division tripartite de la science de l'homme, dans laquelle la physique coexiste avec la physiologie et la psychologie. Les Rapports du physique et du moral (Mémoire de Copenhague) distinguent trois sciences qui ont respectivement pour objet les propriétés des corps bruts, les fonctions des corps vivants, les phénomènes de l'intelligence et de la volonté ${ }^{22}$. Et les Rapports des sciences naturelles vont jusqu'à dire qu'il y a « trois ordres de faits qui servent de fondement à autant de sciences distinctes par leur objet et peut-être aussi par leurs procédés méthodiques $"{ }^{23}$.

On remarquera d'abord que, s'il $y$ a trois ordres de faits et trois sciences, il n'y a pas trois points de vue correspondants, mais toujours deux : « De là deux points de vue de la science de l'homme [...] : celui du naturaliste [...] et celui du psychologiste ", lit-on dans les Rapports des sciences naturelles, quelques pages plus loin ${ }^{24}$; même leçon dans les Rapports du physique et du moral ${ }^{25}$. Par conséquent, il ne saurait être question de faire correspondre la trilogie : physique, physiologie, psychologie, avec la tripartition du Mémoire sur la décomposition de la pensée : aperception immédiate, sentiment immédiat, représentation. La question de savoir quelle science correspond au sentiment immédiat reste entière; elle se complique d'une autre : faut-il admettre la physique, à un titre distinct de la physiologie, au sein de la science de l'homme?

Ce sont les importantes analyses des Rapports du physique et du moral qui permettront d'en décider.

"Les lois de la physique régissent avec constance et uniformité les corps bruts qui leur sont exclusivement soumis ; les lois de la physiologie sont distinctes mais non séparées de celles de la physique dans tout corps organisé vivant, et il a de plus ses lois particulières comme vivant $"{ }^{26}$.

Distinctes mais non séparées : sous cette formulation peu claire, Biran indique que le corps vivant « donne lieu à divers phénomènes purement

22. Rapports du physique et du moral, A, VI, 91.

23. Rapports des sciences naturelles avec la psychologie, A, VIII, 12.

24. Ibid., A, VI, 25.

25. Rapports du physique et du moral, A, VI, 5.

26. Ibid., A, VI, 92. 
physiques ", comme la décomposition de l'air dans les poumons, la réfraction des rayons lumineux dans la vision, etc. ${ }^{27}$. Ces phénomènes, pour s'accomplir dans un organisme vivant, n'en sont pas moins subordonnés aux lois du monde physique; les lois de la physiologie en sont distinctes parce que les phénomènes qu'elles régissent sont distincts de ceux des corps bruts; mais elles n'en sont pas séparées. Toutefois, le vivant, comme tel, a des propriétés et des "lois particulières" : telles sont celles, par exemple, de la contractilité et de la sensibilité. Aucun corps brut ne connaît de tels phénomènes mais on les rencontre dans tous les corps vivants. Et c'est précisément pour avoir reconnu « les lois propres des corps vivants", que Stahl a fondé la physiologie "comme science particulière distincte de la physique ${ }^{28}$.

Il semble que l'on puisse, sur cette base, répondre à la double question : pourquoi la physiologie plutôt que la physique ? Pourquoi pas aussi la physique? Dès lors que la physiologie est reconnue à son titre de science des corps vivants, distincte de la physique, il devient évident qu'elle doit figurer dans la science de l'homme, être pensant et vivant. La présence de la physiologie dans la science de l'homme marque le fait que Biran se situe dans le prolongement de la révolution accomplie par Stahl; son dualisme n'est plus de type cartésien (physique/psychologie), mais de type vitaliste.

Cela ne résout pas la question de la présence de la physique. Faut-il suivre les textes qui placent, à côté de la psychologie, la physiologie et la physique; ou ceux qui englobent la physique dans la physiologie, et ne retiennent que celle-ci comme vis-à-vis de la psychologie? En réalité, il n'y a pas grand inconvénient à suivre les uns ou les autres, indifféremment. La physique et la physiologie correspondent à un seul et même point de vue. Ce sont des sciences diverses, mais cousines; ainsi arrivet-il souvent à Biran de dire de la seconde qu'elle est une «branche » de la première ${ }^{29}$. Selon qu'on est attentif à la distinction des phénomènes du vivant et des phénomènes de la matière inanimée, ou attentif à l'opposition des phénomènes témoignant d'une conscience libre et de ceux témoignant d'un déterminisme strict, on distingue la physique de la physiologie, ou bien l'on oppose la physiologie et la psychologie. Maine de Biran fait l'un et l'autre, sans qu'il y ait là une véritable difficulté. L'essentiel demeure de garder, d'une part, la spécificité de la physiologie par rapport à la physique, d'autre part l'opposition de la physiologie et de la psychologie.

27. Ibid., A, VI, 96.

28. Ibid., A, VI, 92-93.

29. Rapports des sciences naturelles avec la psychologie, A, VIII, 13. 
Reste le problème du «sentiment immédiat » comme point de vue auquel doit normalement correspondre une science. Que faut-il en penser? Et d'abord, est-ce le seul endroit où Maine de Biran procède à une telle division? Nullement. La conclusion du Mémoire sur la décomposition de la pensée retient en effet trois facultés, dont Biran dit qu'elles sont " aussi réellement distinctes entre elles, que le sont les organes particuliers des sens sur qui elles se fondent $"{ }^{30}$. Il s'agit des facultés affective, représentative et aperceptive ou réflexive. La correspondance avec la tripartition des points de vue est aisée : la faculté affective est évidemment ce qui fonde le point de vue du sentiment immédiat, ayant pour objet " tous les éléments sensibles unis dans une même combinaison vivante ". D'autres textes, d'ailleurs, sans les rattacher toutefois à autant de sciences, reprennent la tripartition des points de vue dans des termes assez voisins ${ }^{31}$ pour qu'on soit autorisé à ne pas la considérer comme quelque chose de négligeable. Y a-t-il alors une science qui corresponde à la faculté affective et au point de vue du sentiment immédiat?

Pour répondre à la question, tâchons d'abord d'apporter quelques précisions à cette notion d'un sentiment immédiat, " absolu et général » de tous les éléments sensibles de l'organisme. La correspondance avec Tracy montre d'où vient l'expression : c'est l' « illustre ami » Cabanis qui, dans la « Note [...] touchant le supplice de la guillotine », écrit que « la vie est partout », et que « le moi n'existe que dans la vie générale ${ }^{32}$. Biran, qui commente la formule, ajoute : « Je vois bien là les conditions de l'existence sensitive absolue ${ }^{33}$, mais non point celles de l'existence personnelle. Absolu, chez Biran, est ce qui s'oppose à réflexif et personnel. L'existence sensitive absolue est celle constituée par « les changements opérés dans notre sensibilité », par les « affections directes et simples des parties sentantes ensemble ». Une précision intéressante est donnée dans les lignes suivantes, où Biran note que ce "mode d'existence sensitive [est] complet dans son genre ". Complet dans son genre : on se souvient que c'est le propre d'une science fondée sur un authentique point de vue. Les affections pures, soustraites à la conscience, constitueraient-elles l'objet d'une science autonome? D'autres textes permettent d'avancer dans cette direction.

Les Rapports du physique et du moral évoquent un " tact intérieur », encore appelé « tact immédiat», distingué de la représentation comme

30. Mémoire sur la décomposition de la pensée, A, III, 296.

31. Notes sur le premier probleme de la philosophie, A, XI-3, 207 ; et Essai sur les fondements de la psychologie, T, VIII, 231.

32. Pierre Cabanis, " Note sur l'opinion de MM. CElsmer et Scmmering, et du citoyen Sue, touchant le supplice de la guillotine ", in Euvres philosophiques, Paris, Presses universitaires de France, 1954, t. II, p. 499, note.

33. A Destutt de Tracy, printemps 1804, T, VII, 254. 
de la réflexion, et capable de "pressentir ou deviner immédiatement en nous ", par une " sympathie secrète et naturelle ", les affections pures ${ }^{34}$.

"Ces affections multiples dont la résultante est la vie commune, ne se réfléchissent point dans le sens interne. L'espèce de tact immédiat qui les saisit, ou les devient, n'est point la conscience; car il ne se sait pas, ne s'éclaire pas lui-même " ${ }^{35}$.

"Vie commune ", " vie générale ", " sentiment absolu et général ", ces expressions se correspondent $:$ les deux premières sont assurément l'objet de la troisième.

Que le sentiment, ou tact, immédiat donne bien lieu à une discipline irréductible à la physiologie comme à la psychologie proprement dite, c'est ce qui résulte notamment d'un passage de l'Aperception immédiate, où Biran écrit que les " faits de la nature vivante et simplement sentante " peuvent être observés soit dans le point de vue physiologique soit dans " cette sorte de tact immédiat " qui n'entre point dans le domaine propre de la conscience et reste sur le seuil entre la connaissance purement extérieure et la connaissance vraiment réflexive ${ }^{36}$. Peut-on mettre un nom sur cette discipline et la déterminer plus précisément? Deux textes seront ici utiles. Une page de l'Essai distingue une "psychologie pure ", science de l'esprit et de ce qui lui est propre et inhérent, et une "psychologie mixte », qui « ne considère les faits de l'intelligence que dans leur point de contact avec ceux de la sensibilité " ${ }^{37}$. Et les Rapports du physique et du moral, symétriquement, distinguent une physiologie "spéculative ou hypothétique dans la recherche des causes » et une physiologie « expérimentale ", capable de collaborer à des recherches psychologiques ayant pour objet les affections et les passions ${ }^{38}$. Nommons science mixte cette discipline fondée sur le sentiment immédiat, et constituée par la collaboration de la partie expérimentale de la physiologie et de la partie mixte de la psychologie.

Seule de son espèce, elle implique donc une "double observation", ou " double expérience ", puisqu'elle se sert des faits obtenus par la physiologie et de faits obtenus par l'expérience intérieure. Plus exactement : en tant qu'elle dépend du sentiment immédiat, elle ne peut s'attacher qu'à des faits de l'expérience intérieure. Mais parce que, en la circonstance, ceux-ci sont aussi évanescents qu'Eurydice sous le regard d'Orphée, ils demandent, pour être éclaircis, la collaboration de faits

34. Rapports du physique et du moral, A, VI, 110.

35. Ibid., A, VI, 127-128.

36. De l'Aperception immédiate, A, IV, 169.

37. Essai sur les fondements de la psychologie, T, VIII, 81.

38. Rapports du physique et du moral, A, VI, 89-90. 
recueillis par des sciences objectives, l'anatomie et la physiologie. D'où cette formule :

«Il ne s'agit plus [...] des rapports théoriques que peuvent avoir deux sciences qui diffèrent tant par leurs principes, mais seulement des points homologues en quelque sorte, par lesquels une certaine partie pratique de l'une d'elles peut se rapprocher de telle partie aussi pratique de l'autre $"{ }^{39}$.

Par là, Biran répond, me semble-t-il, à l'objection que l'on ne manquerait pas de faire valoir contre sa théorie d'une discipline «mixte » : la règle d'autonomie des points de vue n'interdit-elle pas, en effet, toute collaboration entre deux sciences? La science mixte ne tâche pas d'expliquer un fait d'une série par un fait d'une autre série, mais de mettre en parallèle les deux faits, d'inscrire entre eux un rapport de condition à conditionné $^{40}$, ou de concomitance ${ }^{41}$. À proprement parler, donc, la science mixte ne donne pas lieu à un troisième ordre de faits; les faits qu'elle embrasse, et que Biran appelle parfois « faits mixtes " ${ }^{42}$, ne sont rien d'autre que des faits intérieurs accolés à des faits extérieurs. Leur association ne crée pas à proprement parler un nouvel ordre de faits.

L'usage fait par Biran de cette science mixte - si tant est que le terme science convienne à une discipline bomée à chercher des rapports entre deux séries hétérogènes de faits - comme aussi les difficultés, tant théoriques que pratiques, qu'elle suscite, requerraient bien d'autres développements. Tenons-nous-en ici au fait lui-même, et concluons donc que la tripartition des points de vue, exposée dans le Mémoire sur la décomposition de la pensée, correspond bien à celle des sciences au sein de la science de l'homme. Celle-ci n'est complète qu'en incluant, à côté d'une physiologie pure, attachée à la connaissance des fonctions du vivant et des mécanismes physiques qui en sont le préalable, à côté d'une psychologie pure aussi, dévolue à l'analyse réflexive des faits de conscience, une "psychologie physiologique ${ }^{43}$ ou science mixte, destinée prioritairement à jeter autant de lumière que possible sur cette partie obscure de l'être vivant et pensant, que sont les affections sans conscience et les phénomènes qui en dépendent. Toujours incertaine, car ne pouvant jouir ni de l'évidence métaphysique propre à la psychologie, ni de l'évidence de fait, propre aux sciences vérifiables, la science mixte est condamnée par sa mixité aux tâtonnements. Mais la science d'un homme

\footnotetext{
39. Ibid., A, VI, 90.

40. Mémoire sur la décomposition de la pensée, A, III, 118.

41. Notes psychologiques, T, XI, 18.

42. Mémoire sur la décomposition de la pensée, A, III, 300 ; Rapports du physique et du moral, A, VI, 90.

43. Rapports du physique et du moral, A, VI, 90.
} 
défini comme "double", vivant et pensant, ne peut prétendre à la complétude et ne peut avancer qu'en l'intégrant en elle. Par RoyerCollard, Lélut, Baillarger et l'école des Annales médico-psychologiques, la leçon sera entendue; et l'automatisme psychologique de Janet devra à la science mixte de Biran plus d'un trait.

François Azouv, Centre national de la recherche scientifique, U.P.R. 75,

7, rue Guy-Mocquet, 94801 Villejuif (janvier 1992). 